\title{
TÉCNICAS DE MINERAÇÃO DE DADOS PARA ANÁLISE DA PRECIPITAÇÃo PLUVIAL DECENAL NO RIO GRANDE DO SUL
}

\section{RAQUEL S. BOSCHI ${ }^{1}$, STANLEY R. DE M. OLIVEIRA ${ }^{2}$, EDUARDO D. ASSAD ${ }^{3}$}

RESUMO: O objetivo deste trabalho foi analisar o comportamento espaçotemporal da precipitação pluvial no Estado do Rio Grande do Sul, entre os decênios de 1987-1996 e 1997-2006, por meio de técnicas de mineração de dados. As séries históricas foram adquiridas no sistema de informações hidrológicas Hidroweb. A metodologia utilizada teve como base o modelo CRISP-DM (Cross Industry Standard Process for Data Mining). Foram definidas áreas pluviometricamente homogêneas para os decênios de 1987-1996 e 1997-2006. Em seguida, pela sobreposição dos agrupamentos obtidos para os dois períodos, encontraram-se seis zonas comuns aos dois decênios (A a F). As alterações ocorridas foram avaliadas nas seguintes escalas temporais: anual, sazonal e mensalmente. Os resultados indicaram incrementos significativos $(20$ a $240 \mathrm{~mm})$ na precipitação anual em todas as zonas, exceto na zona A. Na análise sazonal, as variações foram aleatórias, sendo que, na primavera, todas as zonas apresentaram incremento significativo $(44$ a $142 \mathrm{~mm})$. Na análise mensal, destaca-se a redução ocorrida no mês de janeiro em todas as zonas, exceto na E. Nos demais meses, as variações foram aleatórias. Os resultados mostram que, entre os decênios, houve uma alteração no volume da precipitação pluvial em todas as escalas temporais analisadas.

PALAVRAS-CHAVE: chuva, agrupamento, zonas pluviometricamente homogêneas.

\section{DATA MINING TECHNIQUES FOR DECENNIAL ANALYSIS OF RAINFALL IN RIO GRANDE DO SUL}

\begin{abstract}
The objective of this study was to analyze the spatio-temporal rainfall in Rio Grande do Sul, between 1987-1996 and 1997-2006 decades by using techniques of data mining. The historical series were acquired from the hydrological information Hidroweb system. The methodology used was based on the CRISP-DM (Cross Industry Standard Process for Data Mining) model. First, homogeneous precipitation areas for the 1987-1996 and 1997-2006 decades were defined. Subsequently, by the overlapping of the clusters obtained from both periods, six common areas were defined ranging from A to $\mathrm{F}$. The changes in the volume of precipitation were evaluated annual seasonal and monthly. The results indicated significant increases (20 to $240 \mathrm{~mm}$ ) in annual precipitation in all areas, except in area A. In the seasonal analysis, the variations were random, and in the spring all areas showed significant increase (44 to $142 \mathrm{~mm}$ ). In the monthly analysis, the results revealed a reduction occurred in January in all areas, except in area E. In the other months, the changes were random. The results showed that among the decades there has been a change in the volume of rainfall in all timescales analyzed.
\end{abstract}

KEYWORDS: rain, cluster, homogeneous rainfall areas.

\footnotetext{
${ }^{1}$ Faculdade de Engenharia Agrícola, Universidade Estadual de Campinas (UNICAMP), Campinas - SP, raboschi@yahoo.com.br.

${ }^{2}$ Embrapa Informática Agropecuária, Av. André Tosello, 209, Barão Geraldo, Campinas - SP, stanley@ cnptia.embrapa.br.

${ }^{3}$ Embrapa Informática Agropecuária, Av. André Tosello, 209, Barão Geraldo, Campinas - SP, assad@ @nptia.embrapa.br.

Recebido pelo Conselho Editorial em: 24-6-2010

Aprovado pelo Conselho Editorial em: 22-8-2011
} 


\section{INTRODUÇÃO}

No atual cenário de Aquecimento Global, fenômeno ao qual a ocorrência de secas intensas e eventos extremos de chuva estão cada vez mais associados, o conhecimento do comportamento da precipitação pluvial é fundamental para um planejamento agrícola adequado (IPCC AR 4, 2007).

Em particular, a variabilidade temporal da precipitação observada no Rio Grande do Sul (RS) é apontada como a principal causa nas variações dos rendimentos das principais culturas do Estado (BERLATO \& FONTANA, 1999).

O Estado do RS apresenta uma economia tipicamente agrícola, baseada na produção de grãos e na pecuária. O Estado é responsável por $19 \%$ da produção nacional de grãos, em que a soja e o milho são as duas maiores culturas, correspondendo a aproximadamente $61 \%$ da produção total de grãos do Estado (IBGE, 2009). Esses cultivos são realizados em condições de sequeiro, dependendo, portanto, do regime pluviométrico para o seu desenvolvimento. Em contrapartida, o Estado é responsável por aproximadamente $60 \%$ da produção de arroz do Brasil, que não apresenta essa mesma dependência, uma vez que é irrigado.

Embora as chuvas no Estado do RS sejam bem distribuídas, elas apresentam alta variabilidade interanual, podendo comprometer o rendimento dos cultivos agrícolas. Este fato, associado à imprevisibilidade de suas variações, torna-a o principal fator de risco e insucesso na produção agrícola não irrigada (BERLATO \& FONTANA, 1999; CARGNELUTTI FILHO et al., 2009).

Dentre as técnicas utilizadas para identificar o comportamento da precipitação pluvial, pode-se utilizar a mineração de dados, cujo objetivo é encontrar padrões e tendências nesses dados armazenados (HAN \& KAMBER, 2006). A escolha das técnicas de mineração de dados apresenta-se como uma alternativa promissora, uma vez que essas técnicas podem ser usadas para transformar dados em informações e conhecimentos que irão subsidiar decisões relativas ao planejamento agrícola.

O objetivo consiste em analisar o comportamento espaçotemporal da precipitação pluviométrica decenal no Estado do Rio Grande do Sul, usando as técnicas de mineração de dados, e comparar as possíveis alterações ocorridas entre os decênios de 1987-1996 e 1997-2006.

\section{MATERIAL E MÉTODOS}

A área de estudo compreende o Estado do Rio Grande do Sul situado entre os paralelos $27^{\circ} 03^{\prime} 42^{\prime \prime}$ e $33^{\circ} 45^{\prime} 09^{\prime \prime}$ de latitude sul e entre os meridianos $49^{\circ} 42^{\prime} 41^{\prime \prime}$ e $57^{\circ} 40^{\prime} 57^{\prime \prime}$ de longitude Oeste.

O clima do Estado apresenta dois tipos climáticos conforme a classificação de Köeppen: Cfa (clima temperado úmido com verão quente) e $\mathrm{Cfb}$ (clima temperado úmido com verão temperado). Isto significa que o Estado se situa na faixa de clima subtropical úmido ou temperado com distribuição das chuvas bastante uniforme ao longo do ano, não existindo uma estação seca bem definida. Os totais médios anuais da precipitação pluvial variam de $1.200 \mathrm{~mm}$ a $1.900 \mathrm{~mm}$, podendo ultrapassar os $2.000 \mathrm{~mm}$ em algumas áreas. Segundo BERLATO (1992), a porção sul do Estado apresenta valores de precipitação pluvial inferiores à média do Estado, que se encontra ao redor de $1.550 \mathrm{~mm}$ anuais, enquanto a porção norte apresenta valores superiores a esta média.

A metodologia aplicada neste estudo foi dividida em quatro etapas baseadas no modelo CRISP-DM (CHAPMAN et al., 2000).

A primeira etapa refere-se ao entendimento dos dados. Inicialmente, as séries históricas foram adquiridas na base de dados da Agência Nacional de Água (ANA), no sistema de informações hidrológicas Hidroweb (http://hidroweb.ana.gov.br/) (ANA, 2008). Optou-se por trabalhar com séries homogêneas e contínuas, abrangendo o máximo período amostral possível. Sendo assim, foram selecionadas 79 estações com dados de precipitação pluviométrica diária de 1987 a 2006, cobrindo praticamente todo o estado do RS. 
Optou-se por trabalhar com dados consistidos, uma vez que dados brutos provenientes de estações pluviométricas apresentam problemas devido a erros derivados do próprio processo de coleta e armazenamento. Entre os problemas mais comuns, estão os erros de leitura, de transcrição e digitação, os problemas no aparelho de coleta (danificação, obstrução pela vegetação ou problemas mecânicos no registrador Gráfico) e a ausência de dados durante um determinado período.

Os dados consistidos foram disponibilizados pela Agência Nacional de Água, sendo que a crítica e consistência foram feitas conforme metodologia proposta pela Agência Nacional de Energia Elétrica (ANEEL), baseada no modelo matemático desenvolvido por HOLANDA \& OLIVEIRA (1979).

A segunda etapa é referente à preparação dos dados. A partir dos dados de precipitação pluviométrica diária, construiu-se um banco com dados de chuva mensal, com a finalidade de agrupar as estações em zonas pluviometricamente homogêneas. O conjunto de dados mensais foi dividido em dois conjuntos distintos, em períodos de 10 anos (1987-1996 e 1997-2006). Apenas os dados de chuva foram levados em consideração para a obtenção dos clusters. A normalização dos dados não foi necessária, pois o único atributo considerado foi a chuva mensal, e essa técnica é eficiente quando se utilizam atributos com diferentes ordens de grandeza.

A penúltima etapa trata da modelagem, isto é, da aplicação do algoritmo escolhido na tarefa de mineração de dados. A clusterização (agrupamento) foi a técnica empregada estrategicamente para a segmentação de zonas pluviometricamente homogêneas, para os dois decênios citados anteriormente. O programa computacional utilizado foi o Weka (WITTEN \& FRANK, 2005), e o algoritmo utilizado nas análises foi o k-means (McQUEEN, 1967). O k-means é uma técnica na qual os dados são agrupados de acordo com a métrica de distância euclidiana. O processo fundamenta-se na partição das observações em k-cluster, em que cada observação pertence ao cluster com média mais próxima. Sendo assim, os clusters definidos para o primeiro decênio são independentes daqueles definidos para o segundo decênio.

Foram avaliados os valores de $\mathrm{k}$ de 3 a 7, na tentativa de obtenção do número de cluster mais condizente com a realidade dos dados. A escolha desse intervalo deu-se com base em trabalhos já realizados na obtenção de zonas homogêneas de precipitação pluvial no estado do RS (BECKER et al., 1992; MARQUES et al., 2003; KELLER et al., 2005). Os clusters foram espacializados, em ambiente Spring 4.3, considerando-se a latitude e a longitude de suas respectivas estações, para a visualização dos resultados.

A última etapa refere-se à avaliação dos resultados obtidos. O número de clusters foi definido com base nos resultados obtidos para as duas séries adotadas, por meio da geração de mapas contendo as estações pluviométricas e seus respectivos clusters, assim como por meio da análise da chuva média anual. Nesta etapa do processo, a participação de especialistas do domínio foi fundamental para a escolha do melhor número de zonas homogêneas.

Em seguida, com o intuito de avaliar as alterações ocorridas na precipitação pluvial (incremento ou redução) entre os decênios citados anteriormente, definiram-se, a partir da sobreposição dos clusters dos primeiro e segundo períodos, zonas representativas das regiões comuns aos dois decênios. Verificou-se a existência de seis zonas comuns, denominadas de A-F.

A variabilidade da chuva foi calculada pela diferença entre a precipitação média do período de 1987 a 1996 e a do período de 1997 a 2006, em três granularidades: anual, sazonal e mensal. A avaliação da significância estatística das variações nas médias foi feita por meio da aplicação do Teste Mann-Whitney, ao nível de 5\%. Ainda, com o objetivo de verificar possíveis alterações na distribuição das chuvas ao longo dos meses, fez-se a análise frequencial para períodos mensais. Esta análise foi baseada nos quartis inferior $\left(\mathrm{q}_{25}\right)$, mediano $\left(\mathrm{q}_{50}\right)$ e superior $\left(\mathrm{q}_{75}\right)$. As alterações foram verificadas por meio da comparação das figuras geradas para as duas séries. 


\section{RESULTADOS E DISCUSSÃO}

Foram definidas quatro regiões pluviometricamente homogêneas para o primeiro decênio, denominadas cluster 0 , cluster 1 , cluster 2 e cluster 3 (Figura 1a), e quatro regiões pluviometricamente homogêneas para o segundo decênio, denominadas cluster 4, cluster 5, cluster 6 e cluster 7 (Figura 1b).

(a)

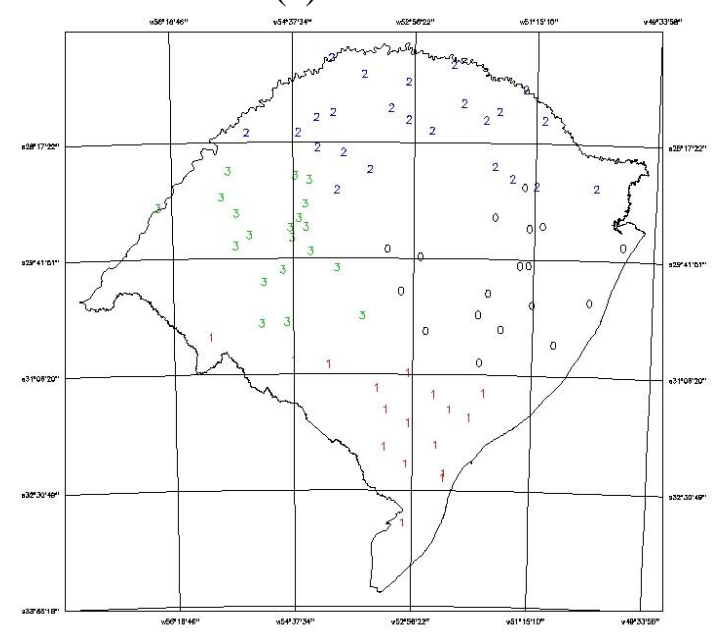

(b)

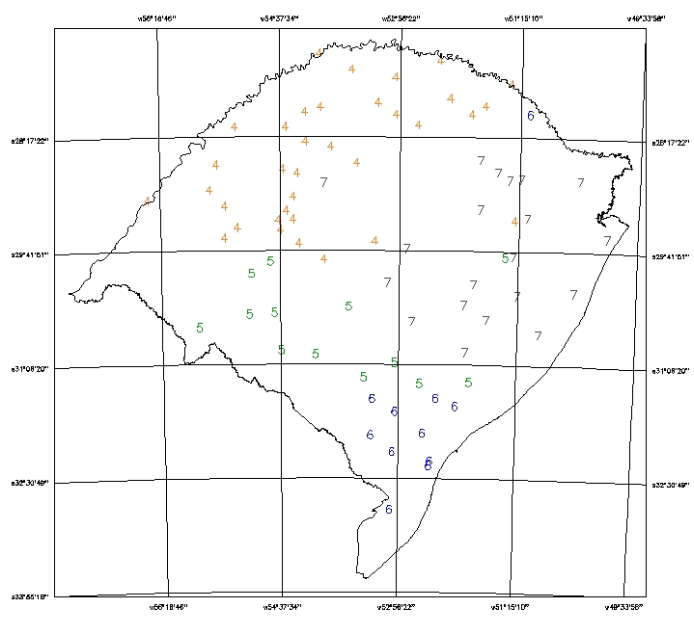

FIGURA 1. Distribuição espacial dos clusters: (a) Período de 1987 a 1996; (b) Período de 1997 a 2006. Spatial distribution of clusters: (a) Period from 1987 to 1996; (b) Period from 1997 to 2006.

A delimitação de zonas pluviometricamente homogêneas para o Estado do RS pode ser observada em diversos trabalhos. Por exemplo, BECKER et al. (1992), a partir de dados mensais de precipitação pluvial no período de 1931 a 1960, delimitaram 4 zonas pluviometricamente homogêneas para o Estado, por meio de um método aglomerativo de hierarquização. MARQUES et al. (2003) utilizaram dados de precipitação mensal de 40 estações pluviométricas do período de 1950 a 1999, definindo-se 7 regiões homogêneas de precipitação pluvial, por meio da técnica de componentes principais. KELLER et al. (2005) utilizaram dados provenientes de 2.341 postos pluviométricos distribuídos por todo o território brasileiro, definindo 6 áreas distintas, com 25 zonas pluviometricamente homogêneas para o Brasil, por meio do método das variâncias mínimas, sendo que, posteriormente, essas regiões foram subdivididas em sub-regiões. Particularmente, na região sul, foram identificadas oito zonas homogêneas, sendo que no Estado do RS foi possível observar seis zonas. Os resultados encontrados neste trabalho coincidem em valor absoluto com aqueles de BECKER et al. (1992), mas diferem dos demais, que utilizam séries mais recentes e metodologias diferentes.

A análise de agrupamentos pode ser observada em diversos outros trabalhos com diferentes finalidades, apresentando, no entanto, um objetivo em comum, que é o estabelecimento de zonas que possam ser consideradas homogêneas. Este tipo de análise visa, na maior parte das vezes, à otimização das análises posteriores (MELO JR et al. 2006; ANDRADE, 1999).

Observando-se as Figuras 1(a) e 1(b), nota-se uma alteração na forma de organização espacial das estações do primeiro para o segundo período. Ou seja, percebe-se um deslocamento, em termos de partição espacial, de algumas estações, sendo alocadas, em clusters distintos, conforme a década estudada.

Na Tabela 1, apresentam-se os volumes de chuva em cada região homogênea. A região sul apresenta os menores volumes de chuva, enquanto os maiores volumes são observados na parte norte do Estado. As regiões centrais apresentam volumes intermediários. 
TABELA 1. Número de estações pluviométricas, localização geográfica e volume de chuva dos oito clusters. Number of rainfall stations, geographical location and rainfall of the eight clusters.

\begin{tabular}{cccc}
\hline Cluster & $\mathrm{N}^{\circ}$ Estações & Localização & Chuva Anual Média $(\mathrm{mm})$ \\
\hline 0 & 18 & Centro-Leste & 1527 \\
1 & 17 & Sul & 1358 \\
2 & 24 & Norte & 1837 \\
3 & 20 & Centro-Oeste & 1563 \\
4 & 35 & Norte/Noroeste & 1845 \\
5 & 13 & Sudoeste & 1630 \\
6 & 11 & Sul & 1538 \\
7 & 20 & Centro-Leste & 1661 \\
\hline
\end{tabular}

Em seguida, a partir da sobreposição dos clusters do primeiro e do segundo períodos estudados, verificou-se a existência de seis zonas comuns aos dois decênios, denominadas de A-F (Figura 2).

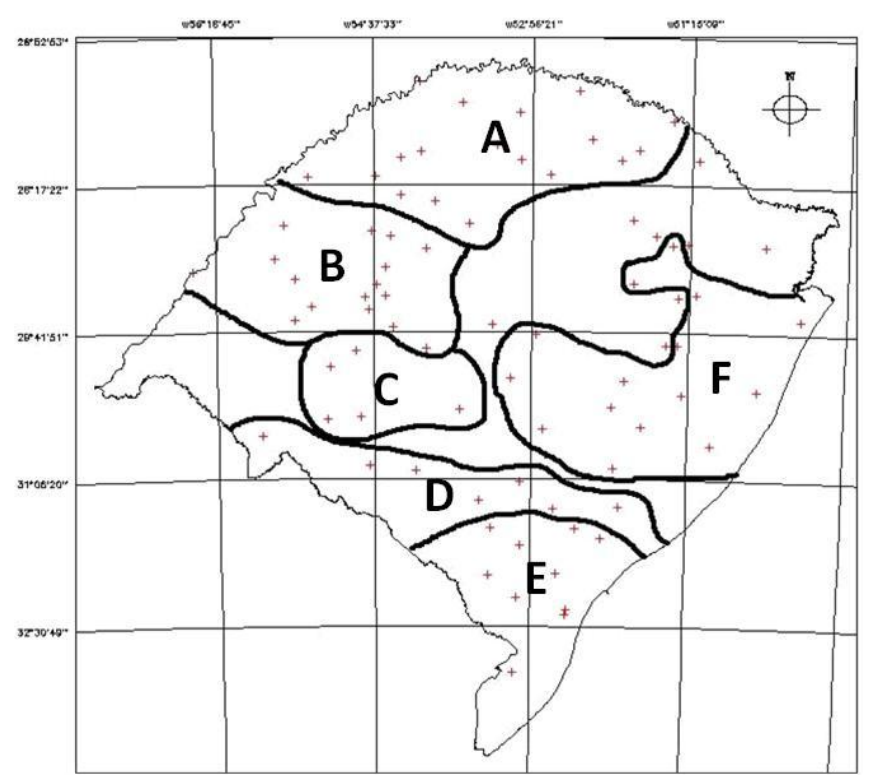

FIGURA 2. Distribuição espacial das zonas comuns aos dois decênios. Spatial distribution of common areas for the two decades.

Na Tabela 2, observa-se a mudança das estações entre o primeiro e o segundo decênios. Por exemplo, a zona A representa a mudança do cluster 2 para o cluster 4 . Em geral, a mudança de cluster reflete a alteração do volume de chuva entre os dois períodos estudados.

TABELA 2. Número de estações pluviométricas por zona de A-F, cluster no primeiro decênio e cluster no segundo decênio. Number of rainfall stations for areas from $A$ to $F$, cluster in the first decade and cluster in the second decade.

\begin{tabular}{cccc}
\hline Zona & $\mathrm{N}^{\circ}$ Estações & Cluster 87-96 & Cluster 97-2006 \\
\hline $\mathrm{A}$ & 18 & 2 & 4 \\
$\mathrm{~B}$ & 15 & 3 & 4 \\
$\mathrm{C}$ & 5 & 3 & 5 \\
$\mathrm{D}$ & 7 & 1 & 5 \\
$\mathrm{E}$ & 10 & 1 & 6 \\
$\mathrm{~F}$ & 15 & 0 & 7 \\
\hline
\end{tabular}


Analisando a Tabela 3, observa-se que os maiores desvios ocorreram na zona E, região onde se concentram os menores volumes de chuva do Estado, enquanto os menores desvios foram observados na zona A, região onde se observam os maiores volumes de chuva. Essas diferenças são, possivelmente, devido à atuação de diferentes fenômenos meteorológicos, assim como das diferenças orográficas entre as regiões.

TABELA 3. Precipitação média anual nos decênios de 87-96 e 97-2006; desvios percentuais e em milímetros entre os decênios; p-valor referente ao teste de Mann-Whitney a $5 \%$. Average annual rainfall in 87-96 and 97-2006 decades; percentage deviations and deviations in millimeters between the decades; p-value referring to the Mann-Whitney test at 5\%.

\begin{tabular}{cccccc}
\hline & & & \multicolumn{3}{c}{ Desvios } \\
\cline { 3 - 4 } Zona & Chuva $87-96(\mathrm{~mm})$ & Chuva $97-2006(\mathrm{~mm})$ & $\%$ & $\mathrm{~mm}$ & p-valor \\
A & 1856 & 1877 & 1,0 & 21,2 & 0,857 \\
B & 1611 & 1816 & 13,0 & 205 & $0^{*}$ \\
C & 1419 & 1642 & 15,7 & 223 & $0,018^{*}$ \\
D & 1464 & 1638 & 12,0 & 174 & $0,038^{*}$ \\
E & 1283 & 1521 & 18,6 & 237,8 & $0^{*}$ \\
F & 1502 & 1595 & 6,0 & 93,2 & $0,041^{*}$ \\
\hline
\end{tabular}

Na Figura 3(a), apresenta-se a espacialização dos desvios em milímetros encontrados para cada uma das zonas. Na Figura 3(b), apresenta-se o mapa com os desvios encontrados para a região Sul do Brasil, pela diferença da chuva acumulada entre as normais de 1961-1990 e 1931-1960 (INMET, 2009). Comparando-se as figuras, notam-se algumas similaridades, como, por exemplo, o não incremento na região norte e os acréscimos nas regiões oeste e leste do Estado.

(a)

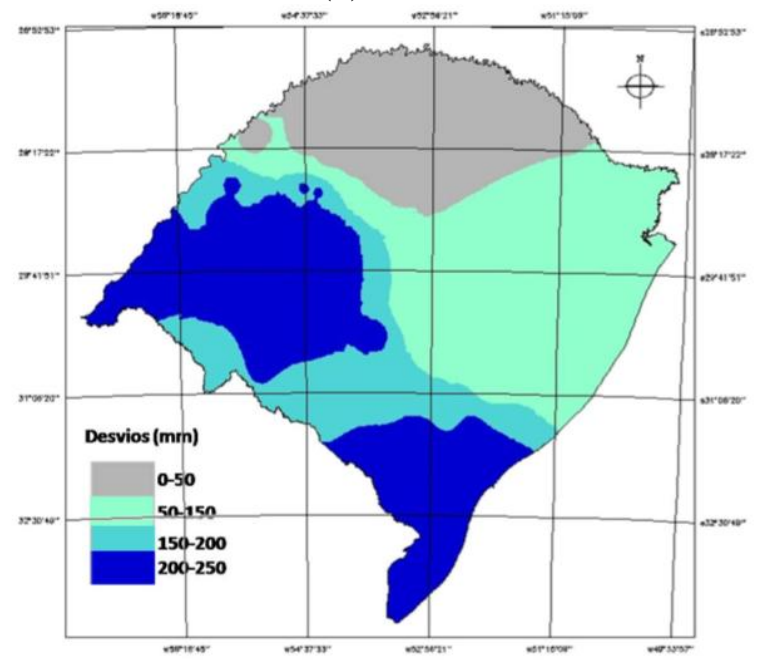

(b)

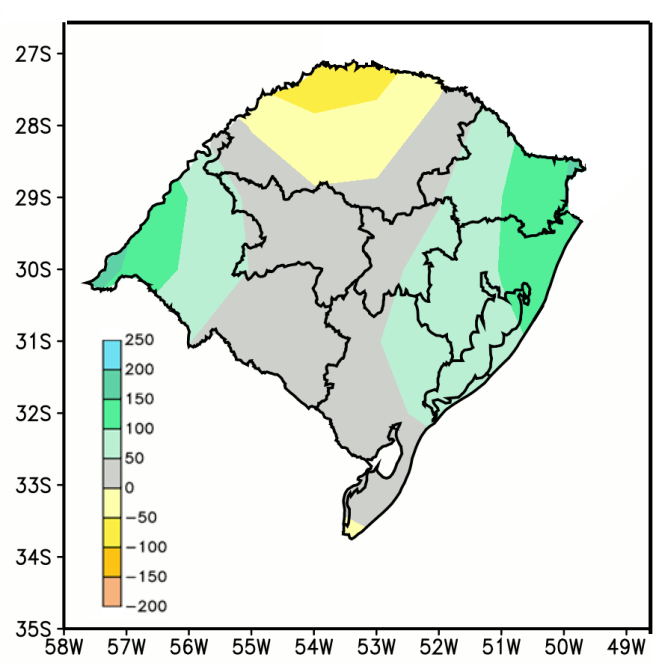

FIGURA 3. (a) Desvios da chuva entre os decênios de 1987-1996 e 1997-2006 para cada uma das zonas; (b) Diferença da chuva acumulada (mm) entre as normais de 1961-1990 e 1931-1960 para a região Sul do Brasil. Fonte: INMET. (a) Deviations of rainfall between the 1987-1996 and 1997-2006 decades for each area, (b) Difference of accumulated precipitation $(\mathrm{mm})$ among the normal from 1961-1990 and 19311960 for the South region of Brazil. Source: INMET.

ÁVILA (1994) comparou as normais climatológicas de 1931-1960 e 1961-1990 e encontrou diferenças significativas na precipitação pluviométrica do Rio Grande do Sul de um período para 
outro, sendo que a normal do segundo período superou a do primeiro em praticamente todo o Estado. VIANA et al. (2006), ao estudarem o comportamento espaçotemporal da precipitação no Rio Grande do Sul entre 1945-1974 e 1975-2004, em 24 estações meteorológicas, observaram um incremento na precipitação anual ao redor de $8 \%$. De fato, tem-se observado recentemente, no sudeste da América do Sul, que inclui a região Sul do Brasil, incremento na precipitação pluvial (HAYLOCK et al., 2006; BARROS et al., 2008).

Embora se tenha observado um incremento na precipitação pluvial em todas as zonas, a exceção foi a zona A. Ao analisar a distribuição das chuvas ao longo dos anos, verificou-se que, nos últimos três anos, houve uma forte redução nos volumes de chuva em todas as zonas (Figura 4)

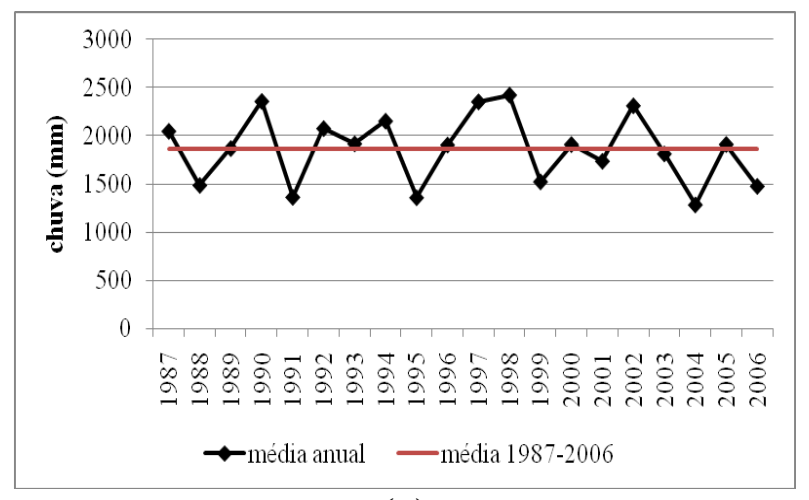

(a)

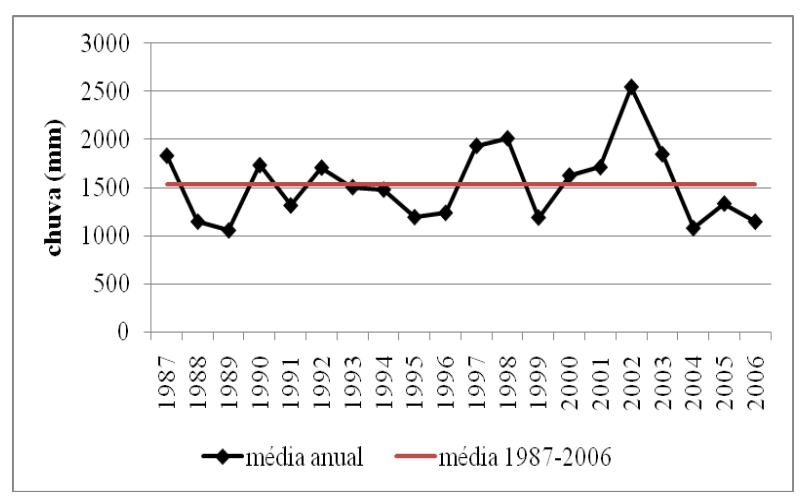

(c)

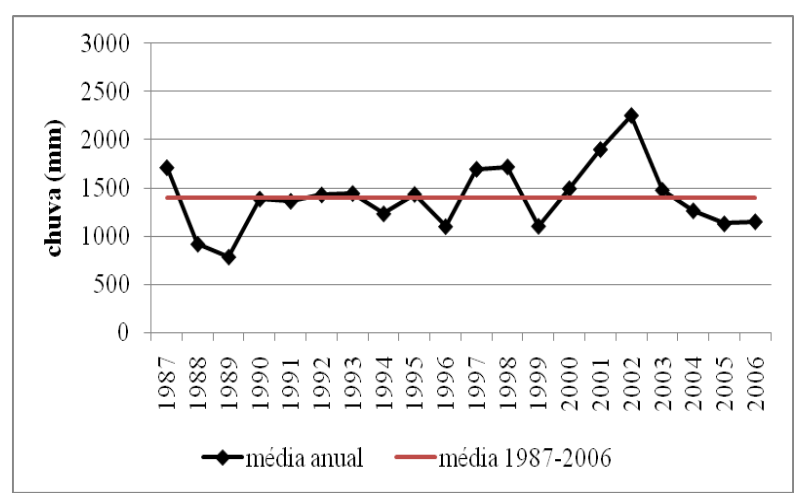

(e)

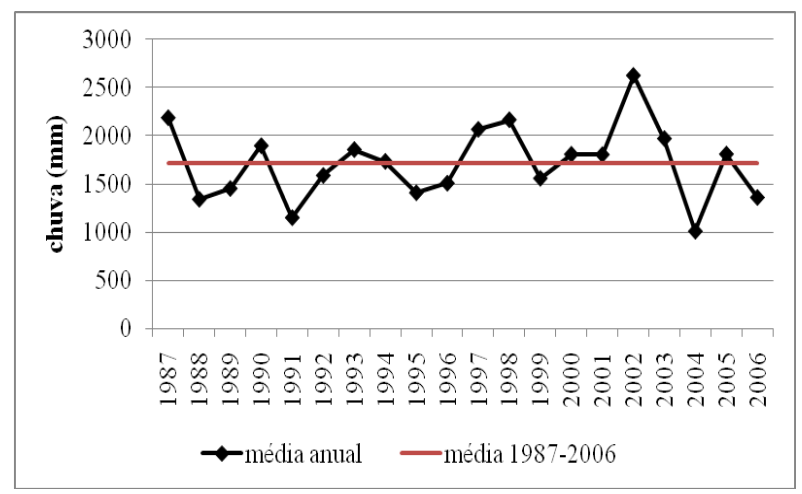

(b)

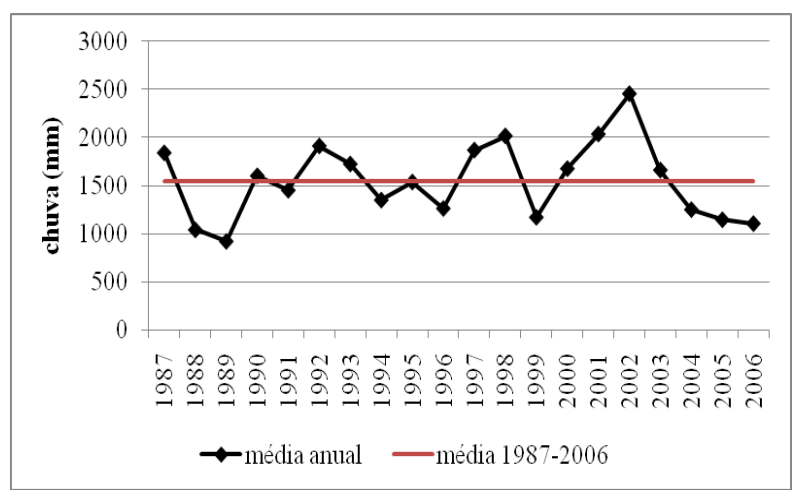

(d)

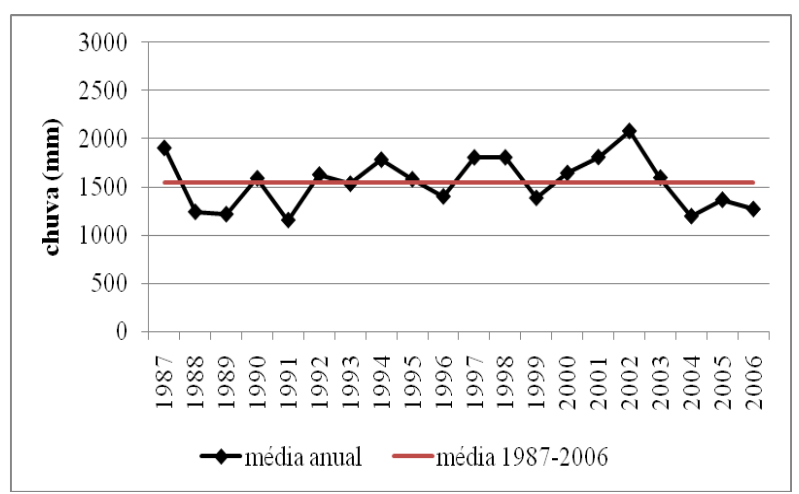

(f)

FIGURA 4. Distribuição da chuva média anual: (a) zona A; (b) zona B; (c) zona C; (d) zona D; (e) zona E; (f) zona F. Distribution of annual average rainfall: (a) area $\mathrm{A}$; (b) area B; (c) area C; (d) area D; (e) area E; (f) area F. 
De acordo com análise apresentada por MARENGO \& VALVERDE (2007), a estiagem ocorrida entre dezembro de 2004 e fevereiro de 2005 foi considerada forte, e o volume de chuva ficou abaixo da normal climatológica.

Por outro lado, no ano de 2002, observaram-se os maiores volumes de chuva, especialmente na zona A (próximo a $2.500 \mathrm{~mm}$ ), região onde se concentram as maiores produções de soja do Estado. Segundo BERLATO \& FONTANA (1999), as maiores produtividades de soja estão fortemente relacionadas com o aumento das chuvas. Diante disso, nota-se, por meio da Figura 4, que o segundo período (1997-2006) apresentou picos mais intensos de chuva, tanto para mais (acima da média), quanto para menos (abaixo da média). Aumentando-se a resolução espacial das análises para o nível sazonal, observa-se que os desvios se comportam de maneira diferente de acordo com a zona estudada (Tabela 4).

TABELA 4. Desvios na precipitação média sazonal entre os decênios de 87-96 e 97-2006 nas seis zonas (A-F). Deviations in seasonal rainfall between the 87-96 and 97-2006 decades in the six areas (A -F).

\begin{tabular}{ccccccccc}
\hline & \multicolumn{8}{c}{ Desvios } \\
\cline { 2 - 9 } & \multicolumn{2}{c}{ Verão } & \multicolumn{1}{c}{ Outono } & \multicolumn{2}{c}{ Inverno } & \multicolumn{2}{c}{ Primavera } \\
\hline Zona & $\%$ & $\mathrm{~mm}$ & $\%$ & $\mathrm{~mm}$ & $\%$ & $\mathrm{~mm}$ & $\%$ & $\mathrm{~mm}$ \\
$\mathrm{~A}$ & 7 & $-32^{*}$ & 4 & $-17,4$ & 5 & $-21,6$ & 15 & $76,4^{*}$ \\
$\mathrm{~B}$ & 3 & 12 & 8 & 36,2 & 0 & $-0,4$ & 34 & $142^{*}$ \\
$\mathrm{C}$ & 4 & 16 & 4 & 15,8 & 14 & $41^{*}$ & 32 & $117,3^{*}$ \\
$\mathrm{D}$ & 4 & $-17 *$ & 21 & $79,6^{*}$ & 7 & 24,6 & 15 & $58,9^{*}$ \\
$\mathrm{E}$ & 12 & 38 & 35 & $112,4^{*}$ & 9,5 & $30,9 *$ & 15 & $43,6^{*}$ \\
$\mathrm{~F}$ & 1 & -5 & 13 & $41,6^{*}$ & 0 & $-4,3$ & 15 & $56,1^{*}$ \\
\hline *ignificativos a 5\% & & & & & & &
\end{tabular}

O verão é o período mais crítico do calendário agrícola do Rio Grande do Sul por coincidir com a época em que as principais culturas de primavera-verão estão na fase de floração e enchimento de grãos, com a maior necessidade de água e máxima sensibilidade ao déficit hídrico. Pode-se dizer, ainda, que durante a primavera e verão, o Rio Grande do Sul apresenta grande demanda evaporativa da atmosfera e, consequentemente, sofre regularmente de deficiências hídricas, mesmo em períodos climatológicos normais.

Diante disso, constata-se que uma queda do nível de chuva pode representar maior limitação dos rendimentos dessas culturas, como a ocorrida na zona A, região onde se concentram as maiores produções de grãos do Estado. Para as culturas de inverno, que representam apenas $10 \%$ da produção total de grãos do Estado, um aumento da precipitação pode favorecer o rendimento desses cultivos. Assim como as demais culturas, as de inverno concentram-se na região da zona A, que apresentou decréscimo nos totais pluviométricos, indicando uma possível diminuição significativa dessas culturas.

Para algumas culturas, como o milho e a soja, tanto o excesso quanto a falta de água no período de germinação são prejudiciais ao seu estabelecimento (SALINAS et al., 1989, citado por FARIAS et al., 2001). Particularmente no caso da soja, excessos hídricos durante esse estádio são mais limitantes que déficits. Dado que no Estado do RS, os volumes de chuva ocorridos durante esta fase são insuficientes para atender às necessidades das culturas de sequeiro (milho e soja), um aumento nos volumes de chuva, como os observados em todas as zonas, pode beneficiar o desenvolvimento vegetativo dessas lavouras. Por outro lado, aumentos de chuvas no início da primavera podem causar prejuízos para as culturas de inverno, pois nesse período essas culturas encontram-se na fase de maturação e colheita.

Estes resultados estão de acordo com VIANA et al. (2006), que observaram que os maiores incrementos na precipitação ocorreram nas estações de transição (primavera e outono). Foram 
encontrados, para todo o Estado, desvios médios de aproximadamente 9\% (primavera) e de 17\% (outono), ao contrário dos resultados obtidos neste trabalho, que foram de $20 \%$ (primavera) e de $13 \%$ (outono).

$\mathrm{Na}$ análise dos dados de precipitação média mensal das duas séries, destaca-se a redução significativa nos volumes de chuva ocorrida durante o mês de janeiro em todas as zonas, exceto na zona $\mathrm{E}$, assim como o aumento ocorrido durante os meses equivalentes ao inverno (Tabela 5).

TABELA 5. Desvios na precipitação média mensal de janeiro, maio, junho e julho entre os decênios de 87-96 e 97-2006, nas seis zonas (A-F). Deviations in monthly mean precipitation in January, May, June and July between the 87-96 and 97-2006 decades in the six $\operatorname{areas}(\mathrm{A}-\mathrm{F})$.

\begin{tabular}{|c|c|c|c|c|c|c|c|c|}
\hline \multirow[b]{3}{*}{ Zona } & \multicolumn{8}{|c|}{ Desvios } \\
\hline & \multicolumn{2}{|c|}{ Janeiro } & \multicolumn{2}{|c|}{ Maio } & \multicolumn{2}{|c|}{ Junho } & \multicolumn{2}{|c|}{ Julho } \\
\hline & $\%$ & $\mathrm{Mm}$ & $\%$ & $\mathrm{~mm}$ & $\%$ & $\mathrm{~mm}$ & $\%$ & $\mathrm{~mm}$ \\
\hline A & 23 & $45^{*}$ & -16 & $-26,1$ & -7 & $-10,4 *$ & -22 & $-33,9 *$ \\
\hline B & 16 & $26,4 *$ & 12 & 13,4 & 17 & $20,4^{*}$ & -23 & $-33,7 *$ \\
\hline $\mathrm{C}$ & 27,5 & $39,1 *$ & 32 & $31,6^{*}$ & 51 & $46,5^{*}$ & -14 & $-17,3$ \\
\hline D & 25 & $35,6 *$ & 51 & $53,5^{*}$ & 51 & $49,1^{*}$ & -27 & $-41,5$ \\
\hline E & 1,8 & 1,9 & 75 & $63,3 *$ & 49 & $43,1^{*}$ & -27 & $-36,8$ \\
\hline $\mathrm{F}$ & 16 & $22,7 *$ & 31 & $29,1 *$ & 0 & $-0,6$ & -6 & $-9,8$ \\
\hline
\end{tabular}

Dado que o mês de janeiro coincide com a fase de floração e enchimento de grãos, importante período para a obtenção de bons rendimentos nas culturas não irrigadas do Estado, e que as zonas $\mathrm{A}$ e B são os locais onde se concentram as maiores plantações de grãos no Rio Grande do Sul, é possível que a redução ocorrida no mês de janeiro tenha prejudicado o início da floração e o enchimento de grãos das lavouras da porção norte e noroeste do Rio Grade do Sul, propiciando perdas de produtividade.

Incrementos nos meses de maio e junho representam um ponto positivo para os cultivos de inverno na região, como por exemplo, o trigo. Os aumentos observados durante os meses equivalentes ao início de plantio dos cultivos de primavera-verão (outubro, novembro e dezembro) podem ter favorecido o estabelecimento dessas culturas (Tabela 6).

TABELA 6. Desvios na precipitação média mensal de outubro, novembro e dezembro entre os decênios de 87-96 e 97-2006, nas seis zonas (A-F). Deviations in the average monthly rainfall for October, November and December between the 87-96 and 97-2006 decades in the six areas (A-F).

\begin{tabular}{crrrrrc}
\hline & \multicolumn{9}{c}{ Desvios } \\
\cline { 2 - 7 } & \multicolumn{7}{c}{ outubro } & \multicolumn{3}{c}{ novembro } & \multicolumn{2}{c}{ dezembro } \\
\hline Zona & $\%$ & $\mathrm{~mm}$ & $\%$ & $\mathrm{~mm}$ & $\%$ & $\mathrm{~mm}$ \\
$\mathrm{~A}$ & 37 & $73,5^{*}$ & 17 & $23,5^{*}$ & 21 & $31,3^{*}$ \\
$\mathrm{~B}$ & 74 & $107,8^{*}$ & 19 & $24,1^{*}$ & 53 & $54,2^{*}$ \\
$\mathrm{C}$ & 43 & $54,8^{*}$ & 43 & $42,9^{*}$ & 45 & 47 \\
$\mathrm{D}$ & 5 & 7,4 & 8 & 8,5 & 26 & 29,2 \\
$\mathrm{E}$ & 8 & 8,8 & -10 & $-11,4$ & 55 & $46,7^{*}$ \\
$\mathrm{~F}$ & 26 & $36,3^{*}$ & 10 & 11,5 & 20 & 21,8 \\
\hline
\end{tabular}

*significativos a $5 \%$

Ainda, na análise frequencial da precipitação pluvial mensal, observou-se a distribuição das chuvas ao longo dos meses, para os períodos de 1987 a 1996 e de 1997 a 2006, para anos normais 
(mediana $-\mathrm{q}_{50}$ ), anos secos (quartil inferior $-\mathrm{q}_{25}$ ) e anos chuvosos (quartil superior $-\mathrm{q}_{75}$ ) nas seis zonas de A-F (Figura 5).

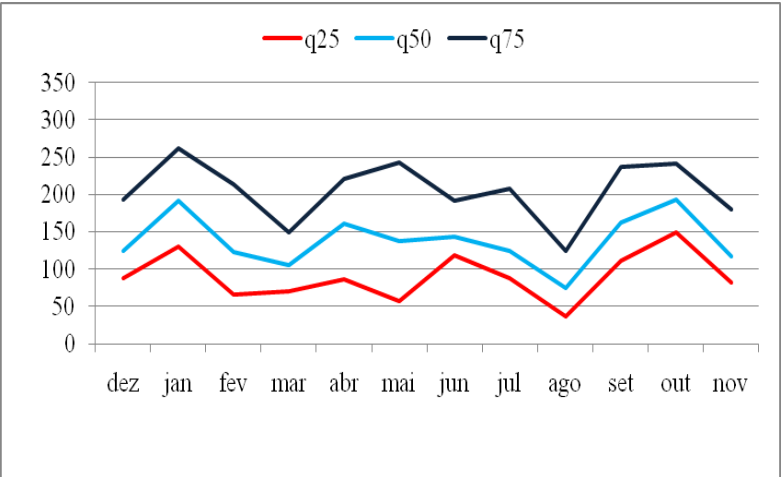

(a)

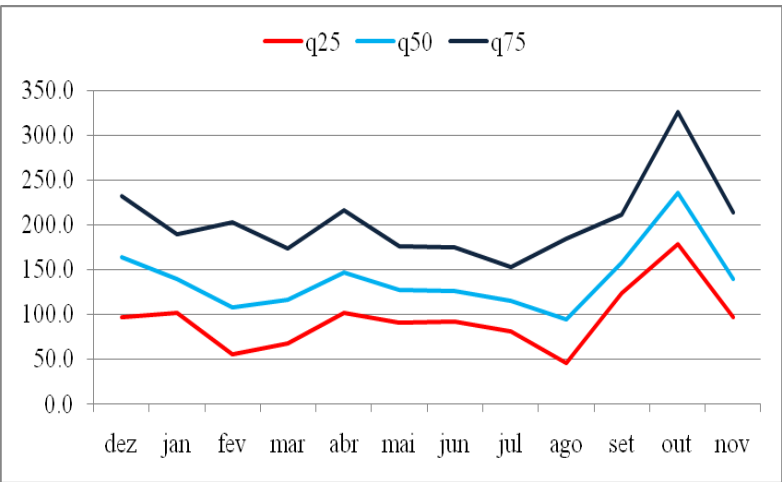

(b)

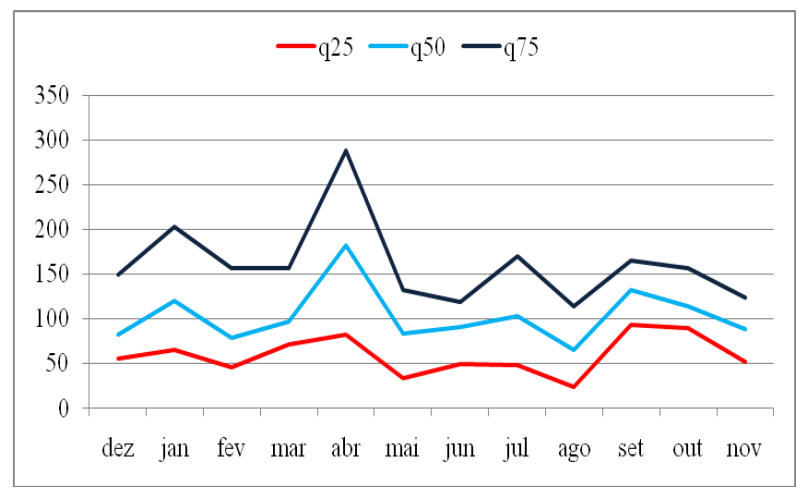

(e)

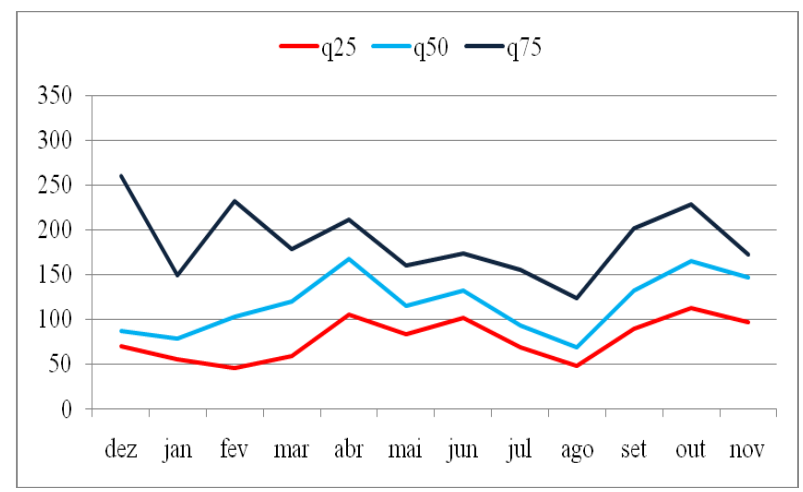

(f)

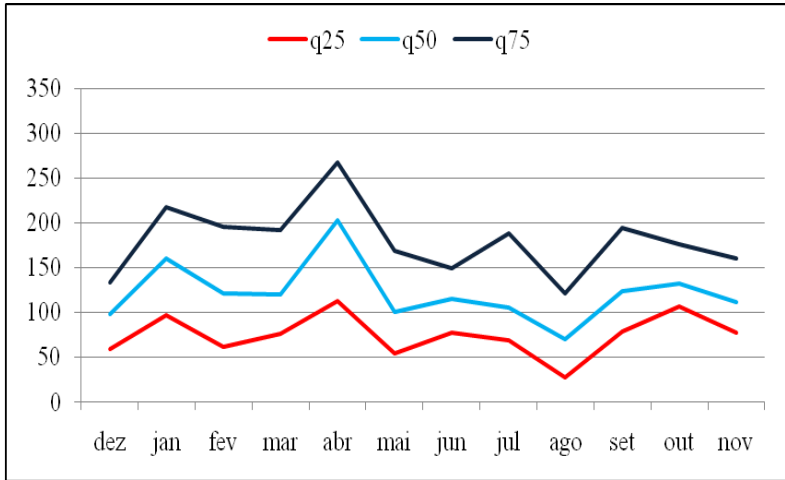

(c)

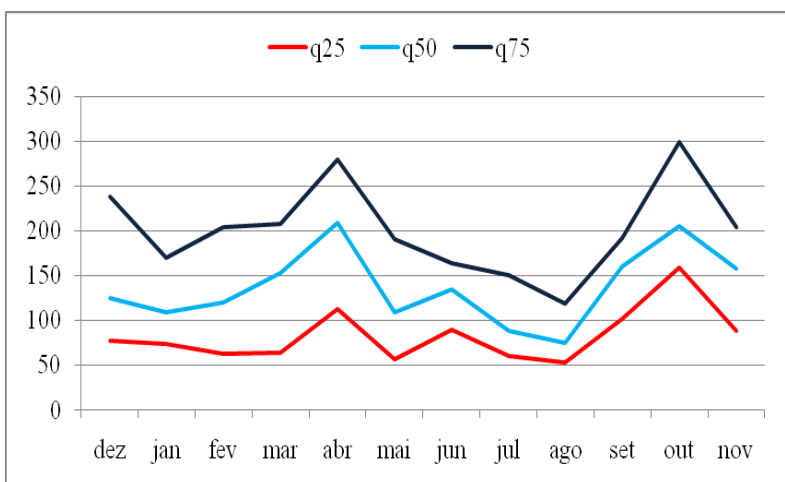

(d)

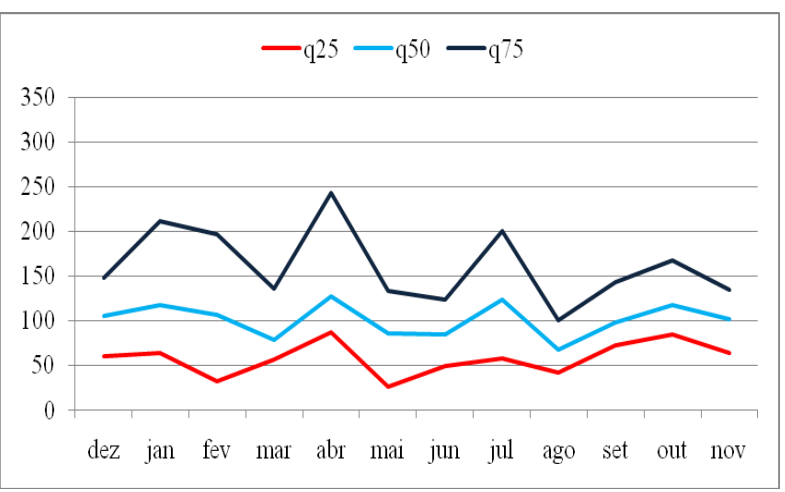

(g)

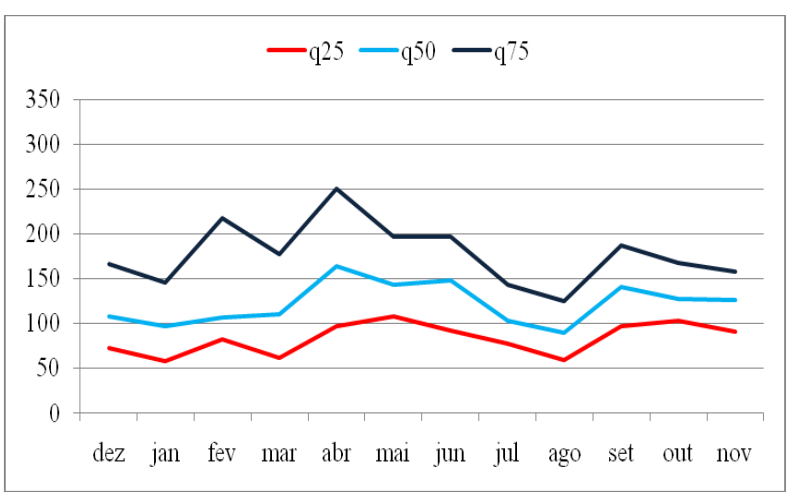

(h) 


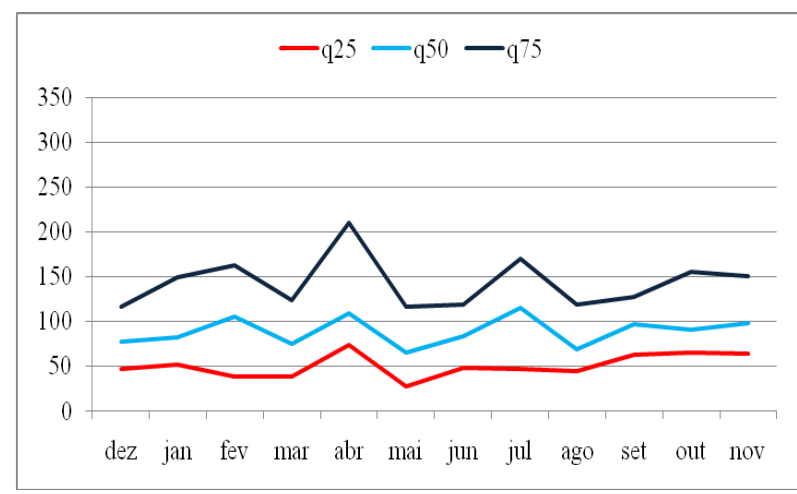

(i)

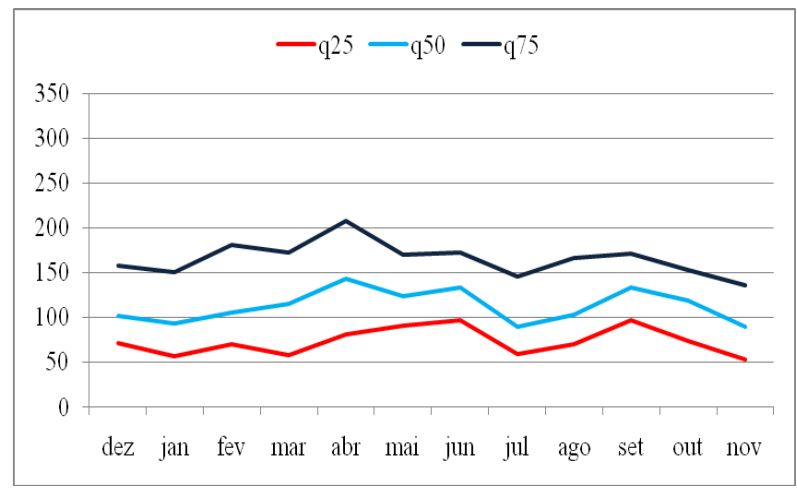

(j)

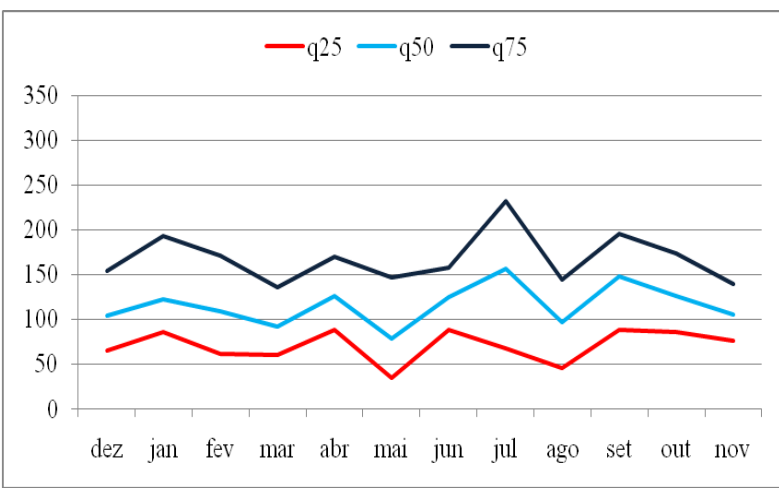

(k)

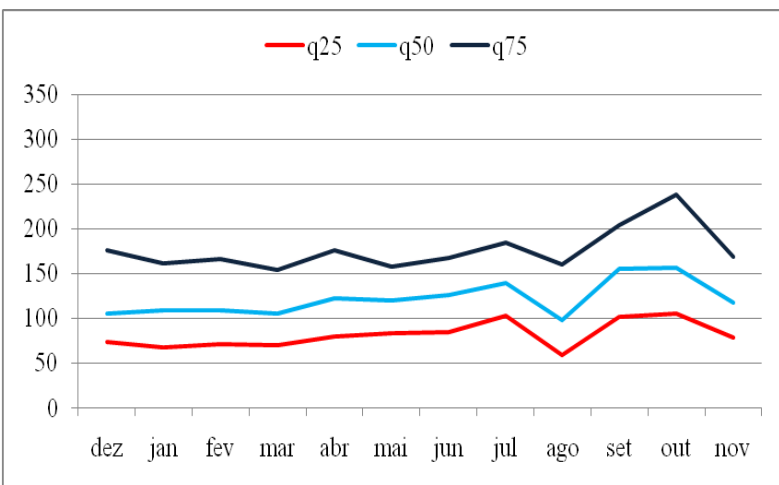

(1)

FIGURA 5. Análise frequencial: (a) zona A de 1987-1996; (b) zona A de 1997-2006; (c) zona B de 1987-1996; (d) zona B de 1997-2006; (e) zona C de 1987-1996; (f) zona C de 19972006; (g) zona D de 1987-1996; (h) zona D de 1997-2006; (i) zona E de 1987-1996; (j) zona E de 1997-2006; (k) zona F de 1987-1996; (l) zona F de 1997-2006. Frequencial analysis: (a) area A from 1987 to 1996; (b) area A from 1997 to 2006; (c) area B from 1987 to 1996; (d) area B from 1997 to 2006; (e) area C from 1987 to 1996 (f) area C from 1997 to 2006; (g) area D from 1987 to 1996; (h) area D from 1997 to 2006; (i) area $E$ from 1987 to 1996; (j) area $E$ from 1997 to 2006; (k) Frequencial area F from 1987 to 1996; (l) area F from 1997 to 2006.

De modo geral, observa-se que o segundo período apresentou um aumento no volume de chuvas em praticamente todos os meses, para todas as frequências, com exceção do mês de janeiro. Ainda, notou-se uma suavização das curvas no segundo período, especialmente durante os meses de maio, junho e julho. Picos durante o mês de outubro também foram mais frequentes durante o segundo decênio.

\section{CONCLUSÕES}

A técnica de agrupamento apresenta eficiência na análise do comportamento espaçotemporal da precipitação pluviométrica no Estado do Rio Grande do Sul.

A precipitação pluvial anual apresentou um incremento significativo $(20-240 \mathrm{~mm})$ entre os decênios de 1987-1996 e 1997-2006, em todas as zonas, exceto na zona A. Os maiores desvios percentuais ocorreram na região E $(18,6 \%)$, localizada ao sul do Estado, e os menores, na zona A $(1,1 \%)$, localizada ao norte do Estado.

O segundo período (1997-2006) apresentou picos mais intensos de chuva, tanto para mais (acima da média), quanto para menos (abaixo da média), sendo que os menores volumes foram observados especialmente nos últimos três anos da série (2004, 2005 e 2006). 
As alterações observadas de precipitação pluvial sazonal não apresentaram padrão de redução ou incremento, ocorrendo variações entre classes e períodos.;

No segundo decênio, todas as zonas apresentaram redução significativa na precipitação pluvial média do mês de janeiro, com exceção da zona E. Os volumes variaram de $45 \mathrm{~mm}$, ocorridos zona A, a $23 \mathrm{~mm}$ ocorridos na zona F.

Por fim, como trabalho futuro, sugere-se:

Relacionar as mudanças encontradas com dados de produção/produtividade para verificar os reais impactos na agricultura do Estado.

Estudar as possíveis causas que influenciaram nas variações de precipitação pluvial observadas neste estudo, considerando as escalas temporais: anual, sazonal e mensalmente.

Acrescentar outras variáveis ao conjunto de dados, tais como temperatura e umidade, para verificar possíveis associações com os fenômenos estudados.

\section{AGRADECIMENTOS}

À CAPES, pelo apoio financeiro. Aos colegas Giampaolo Queiroz Pellegrino, Ana Maria Heuminski de Ávila, Fernanda Ultremare e Cristiano Amâncio, pelo apoio e colaboração.

\section{REFERÊNCIAS}

AGÊNCIA NACIONAL DE ÁGUA. HIDROWEB: Dados Hidrológicos. Disponível em: <http://hidroweb.ana.gov.br/>. Acesso em: jun. 2008.

ANDRADE, E.M. Regionalization of small watersheds in arid and semiarid regions: cluster and Andrews' curve approaches. Engenharia Agrícola, Jaboticabal, v.18, n.4, p.39-52, 1999.

ÁVILA, A.M.H. Regime de precipitação pluvial no Estado do Rio Grande do Sul com base em séries de longo prazo. 1994. 87 f. Dissertação (Mestrado em Agronomia) - Universidade Federal do Rio Grande do Sul, Porto Alegre, 1994.

BARROS, V.B.; DOYLE, M.E.; CAMILLONI, I.A. Precipitation Trends in Southeastern South America: relationship with ENSO phases and with low-level circulations. Theoretical and Applied Climatology, Viena, v.93, n.1-2, p.19-33, 2008.

BECKER, C.T.; BRAGA, C.C.; CEBALLOS, J.C. Regionalização da precipitação e temperatura no Estado do Rio Grande do Sul a partir da análise de agrupamento. In: CONGRESSO BRASILEIRO DE METEOROLOGIA, 7., 1992, São Paulo. Anais... São Paulo: SBMet, 1992. p.225-229.

BERLATO, M.A. As condições de precipitação pluvial no Estado do Rio Grande do Sul e os impactos das estiagens na produção agrícola. In: BERGAMASCHI, H.; BERLATO, M.A.; FONTANA, D.C.; CUNHA, G.R.; SANTOS, M.L.V. dos; FARIAS, J.R.B.; BARNI, N.A. Agrometeorologia aplicada à irrigação. Porto Alegre: UFRGS, 1992. p.11-23.

BERLATO, M.A.; FONTANA, D.C. Variabilidade interanual da precipitação pluvial e rendimento da soja no Estado do Rio Grande do Sul. Revista Brasileira de Agrometeorologia, Santa Maria, v.7, n.1, p.119-125, 1999.

CARGNELUTTI FILHO, A.; MATZENAUER, R.; MALUF, J. R. T.; RADIN, B. Variabilidade temporal e espacial da precisão das estimativas de elementos meteorológicos no Rio Grande do Sul. Ciência Rural, Santa Maria, v.39, n.4, p.962-970, 2009.

CHAPMAN, P.; CLINTON, J.; KERBER, R.; KHABAZA, T.; REINARTZ, T.; SHEARER, C.; WIRTH, R. CRISP-DM 1.0: step-by-step data mining guide. Illinois: SPSS, 2000. 78 p. Disponível em: <http://www.crisp-dm.org/CRISPWP-0800.pdf>. Acesso em: 02 jun. 2008. 
FARIAS, J.R.B.; ASSAD, E.D.; ALMEIDA, I.R.; EVANGELISTA, B.A.; LAZAROTTO, C.; NEUMAIER, N.; NEPOMUCENO, A.L. Caracterização de risco de déficit hídrico nas regiões produtoras de soja no Brasil. Revista Brasileira de Agrometeorologia, Passo Fundo, v.9, n.3, p.415421, 2001.

HAN, J.; KAMBER, M. Data mining: concepts and techniques. $2^{\text {nd }}$ ed. San Francisco: Morgan Kaufmann Publishers, 2006. p.770.

HAYLOCK, M.R.; PETERSON, T.; ABREU DE SOUSA, J.R.; ALVES, LM.; AMBRIZZI, T.; BAEZ, J.; BARBOSA DE BRITO, J. I.; BARROS, V.R.; BERLATO, M.A.; BIDEGAIN, N.M.; CORONEL, G.; CORRADI, V.; GRIMM, A.M.; DOS SANTOS, R.J.; KAROLY, D.; MARENGO, J.A.; MARINO, M.B.; MEIRA, P.R.; MIRANDA, G.C.; MOLION, L.; MUNCUNIL, D.F.; NECHET, D.; ONTANEDA, G.; QUINTANA, J.; RAMIREZ, E.; REBELLO, E.; RUSTICUCCI, M.; SANTOS, J.L.; VARILLAS, I.T.; VILLANUEVA, J.G.; VINCENT, L.; YUMICO, M. Trends in total and extreme South American rainfall 1960-2000 and links with sea surface temperature. Journal of Climate, Boston, v.19, n.8, p.1.490-1.512, 2006.

HOLANDA, C.V.M.; OLIVEIRA, E. Programa para Homogeneização de Dados - PROHD. In: SIMPÓSIO DE HIDROLOGIA, 3., 1979, Brasília. Anais... Porto Alegre: Associação Brasileira de Recursos Hídricos, 1979. p.810-845.

IBGE - INSTITUTO BRASILEIRO DE GEOGRAFIA E ESTATÍSTICA. SIDRA: agricultura. Disponível em: < http://www.sidra.ibge.gov.br >. Acesso em: 02 mar. 2009.

INMET: Climatologias (1931-60) (1961-90). Trabalho elaborado pela CDP/INMET, 2009.

INTERGOVERNMENTAL PANEL ON CLIMATE CHANGES IPCC. Climate Change 2007: summary for policymakers, Geneva, 2007.

KELLER FILHO, T.; ASSAD, E.D.; LIMA, P.R.S.R. Regiões pluviometricamente homogêneas no Brasil. Pesquisa Agropecuária Brasileira, Brasília, v.40, n.4, p.311-322, 2005.

MARENGO, J.A.; VALVERDE, M.C. Caracterização do clima no Século XX e Cenário de Mudanças de clima para o Brasil no Século XXI, usando os modelos do IPCC-AR4. Revista Multiciência, Campinas, v.8, p.5-28, 2007.

MARQUES, J.R.; BERLATO, M.; FONTANA, D. Regiões homogêneas de precipitação pluvial mensal segundo o comportamento temporal sobre o Rio Grande do Sul. In: CONGRESSO BRASILEIRO DE AGROMETEOROLOGIA, 13., 2003, Santa Maria. Anais... Santa Maria: SBA, 2003, v.2, p.1.057-1.058.

McQUEEN, J.B. Some methods for classification and analysis of multivariate observations. In: BERKELEY SYMPOSIUM ON MATHEMATICAL STATISTIC AND PROBABILITY, 7., 1967, Berkeley. Proceedings... p.281-297.

MELO JUNIOR, J.C.F.; SEDIYAMA, G.C.; FERREIRA, P.A.; LEAL, B.G. Determinação de regiões homogêneas quanto à distribuição de frequência de chuvas no leste do Estado de Minas Gerais. Revista Brasileira de Engenharia Agrícola e Ambiental, Campinas Grande, v.10, n.2, p.408416, 2006.

VIANA, D.R.; AQUINO, F.E.; MATZENAUER, R. Comportamento espaçotemporal da precipitação no Rio Grande do Sul entre 1945-1974 e 1975-2004. In: CONGRESSO BRASILEIRO DE AGROMETEOROLOGIA, 14., 2006, Florianópolis. Anais... Florianópolis: SBMET, 2006. 1 CD-ROM.

WITTEN, I.H.; FRANK, E. Data mining: practical machine learning tools and techniques. 2.ed. San Francisco: Morgan Kaufmann, 2005. 52p. 